\title{
Alterations of antioxidant status markers in dairy cows during lactation and in
}

the dry period

A. Omidi ${ }^{1, a}$, M. H. Fathi ${ }^{2}$ and M. O. Parker ${ }^{3}$

${ }^{1}$ Department of Animal Health Management, School of Veterinary Medicine, Shiraz

University, Shiraz, Iran

${ }^{2}$ Department of Animal Sciences, Agriculture Faculty, University of Birjand, Birjand, Iran

${ }^{3}$ School of Health Sciences and Social Work, University of Portsmouth, Portsmouth, UK

aCorresponding author: Arash/Omidi. Email: aomidi@shirazu.ac.ir

Short title: Antioxidant status in blood of dairy cows

\section{Abstract}

The purpose of the study was to evaluate alterations in concentration of total antioxidant capacity (TAC) of plasma, and plasma total thiols as markers of oxidative protein damage, and malondialdehyde (as a final product of lipid peroxidation, in samples obtained at different times of lactation cycle and dry period of dairy cows. We found that TAC was significantly lower in the primiparous cows compared to multiparous cows. This study clearly demonstrates a need for monitoring primiparous cows during the production cycle, especially when they faced with severe metabolic conditions. Furthermore, TAC may be a sensitive, reliable and useful indicator for measurement of cumulative effects of antioxidants as an addition to metabolic profile tests, which are currently used to analyse dairy cattle health.

Keywords: antioxidant status, dairy cows, dry period, lactation cycle 


\section{Introduction}

Evaluation of blood biochemical parameters is critical to identify and monitor health status and disease incidence in dairy cattle farms. Evaluation of oxidative status is a relatively young field in ruminant medicine. Indeed, most studies in cattle, sheep and goats have been sporadic and dealt predominantly with the effects of diseases, including mastitis, pneumonia, sepsis, acidosis, ketosis, enteritis, joint diseases and retained placenta (Celi, 2010). Increasingly, research has focused on metabolic diseases that affect dairy ruminants during the peripartum period (LeBlanc, 2010; Sepúlveda-Varas et al. 2013). Exploration of metabolic profiles through the analysis of biochemical parameters in dairy cattle blood is well established (e.g., Payne et al. 1970). However, metabolic profile tests as an applicable approach can reveal which areas of nutrition and management require more attention in dairy herds (Kolver \& Macmillan, 1994; Macrae et al. 2006).

In recent years, there has been a growing concern for detection of free radical damage as a complementary tool in the evaluation of metabolic status. Under normal physiological conditions, the body has sufficient reserves of antioxidants to combat free radical accumulation. Production of reactive oxygen species (ROS) is the outcome of aerobic metabolic pathways in the body (Halliwell, 2006). Achieving the balance between production and neutralization of ROS is crucial for maintaining the homeostasis (Poljsak et al. 2013). Oxygen free radicals are produced during the productive phases of domestic animals (Piccione et al. 2006; Celi, 2010). Oxidative stress results from increased exposure to, or production of, oxidants, and can be monitored with a number of biomarkers. Several methods have been developed to assess total antioxidant capacity (TAC; Del Rio et al. 2005); TAC, for example, describes the dynamic equilibrium between prooxidants and antioxidants in the 
plasma compartment (Castillo et al. 2005). Monitoring of the efficiency of antioxidative systems against ROS is made by the estimation of TAC. The primary antioxidant capacity of serum is derived from non-enzymatic antioxidants, such as glutathione, $\alpha$-tocopherol, $\beta$-carotene and uric acid (Halliwell \& Gutteridge, 1990). A sensitive, reliable and useful indicator for measurement of cumulative action of all the antioxidants present in plasma is TAC. TAC is also a useful tool for measuring stress in transported calves (Pregel et al. 2005), and could potentially be used to evaluate the general nutritional status of animals (Mandebvu et al. 2003; Zdunczyk et al. 2006).

Evaluation of TAC along with measuring thiols groups as the major portion of the total body antioxidants, and plasma levels of malondialdehyde (MDA), a degradation product of lipid peroxidation are useful in specify their relationship with different lactational stage and dry period in dairy cow.

Plasma total thiols as marker of oxidative protein damage constitute the major portion of the total body antioxidants and play a significant role in defence against reactive oxygen species. The antioxidant capacity of thiol compounds is due to the sulphur atom, which can easily accommodate the loss of a single electron (Karoui et al. 1996). Decreased levels of total thiols are reported in various physiological and pathological conditions, including diabetes mellitus, cardiovascular disorders, and disorders related to kidney, neurological disorders due to excess free radical generation (Mongolia et al. 2009). The entire antioxidant system seems to be under homeostatic control

It is clear that primiparous cows, and cows in early lactation, are more sensitive than multiparous cows to disease (metabolic \& infectious disease). 
Therefore, the aim of this current study was to carry out an assessment of metabolic and oxidative markers in order to find a sensitive test that revealed the health condition of at-risk cows (especially primiparous or in early lactation). This was conducted using three parameters: (1) TAC; (2) total thiols, as markers of oxidative protein damage, and; (3) plasma levels of MDA.

\section{Material and methods}

Animals, husbandry and nutrition

A total of 69 Holstein breed dairy cows were used in the study, and each animal was observed once. All animals were held in intensive farm breeding premises, divided into groups according to level of production. Feed was adjusted to production intensity, and consisted of alfalfa hay, corn silage, wheat bran and concentrates with energy and protein supplements (Table 1 and 2). For each animal the clinical history was reviewed with the farm manager, physical examinations were made by the first author. Cows with suspected clinical disease were excluded from the study and an alternate animal chosen from the list previously selected by random numbers. Cows were body condition scored on a five-point scale ( $1=$ thin, $5=$ obese). Maximum 0.25 differences in body condition score (BCS) were accepted between cows in each group. Group allocation was as thus: Group E (fresh): dairy cows from the 3th to 21th day of lactation, (E1: primiparous, E2: multiparous). Group M (peak milk): dairy cows from the 45th to 100th day of lactation, (M1: primiparous, M2: multiparous). Group L: dairy cows from the 260th day of lactation until the end of milk production, (L1: primiparous, L2: multiparous). Group F: dairy cows in the far-off dry period (from the 305th -345th day). Group C: dairy cows in the close-up dry period (from the 346th360th day). 


\section{Sample collection and analytical procedures}

Blood samples were collected by coccygeal venepuncture between 8 and 9 A.M. on three consecutive days. Samples were collected in plain tubes and vacationers containing EDTA (10:1 ratio), centrifuged (750g, 15 minutes, room temperature), and stored at $-80 \mathrm{C}$ until analysis. The samples with haemolysis were discarded.

\section{Measurement of TAC}

The ferric reducing antioxidant potential (FRAP) assay was used to measure TAC, using the DetectX® Ferric Reducing Antioxidant Power (FRAP ${ }^{\mathrm{TM}}$ ) Detection Kit (Company Arbor Assays, USA). FRAP is expressed as mmol/L.

\section{Measurement of total thiols}

Total thiols in plasma were determined according to the spectrometric method of $\mathrm{Hu}$ using the Measure-iT ${ }^{\mathrm{TM}}$ Thiol Assay Kit (Invitrogen European Headquarters Company, Paisley PA4 9RF, UK). Plasma total thiols are expressed as mmol/L ( $\mathrm{Hu}$, 1994).

\section{Measurement of MDA}

To evaluate lipid peroxidation in serum, a modified HPLC method was used based on the reaction of MDA with thiobarbituric acid (TBA), to form a coloured MDA-TBA adduct (Lykkesfeldt, 2001). Forty $\mu \mathrm{L}$ of sample was diluted with $100 \mu \mathrm{L}$ of $\mathrm{H}_{2} \mathrm{O}$ and mixed with $20 \mu \mathrm{L}$ of $2.8 \mathrm{mmol} / \mathrm{L}$ butylated hydroxyl toluene (BHT) in ethanol, $40 \mu \mathrm{L}$ of $81 \mathrm{~g} / \mathrm{L}$ sodium dodecyl sulphate and $600 \mu \mathrm{L}$ of TBA reagent $(8 \mathrm{~g} / \mathrm{L}$ TBA diluted $1: 1$ with $200 \mathrm{ml} / \mathrm{L}$ acetic acid adjusted to $\mathrm{pH} 3.5$ with $\mathrm{NaOH}$ ). The mixture was immediately heated $\left(60 \mathrm{~min}\right.$ at $\left.95^{\circ} \mathrm{C}\right)$ and cooled with running water; $200 \mu \mathrm{L}$ of $\mathrm{H}_{2} \mathrm{O}$ and $1000 \mu \mathrm{L}$ of butanol-pyridine $(15: 1, \mathrm{v} / \mathrm{v})$ were then added. After vigorous mixing, the organic layer was separated by centrifugation ( 3 minutes at $16,000 \mathrm{~g}$ ). The supernatant was analysed on a UV-visible spectrophotometer fitted with an $80 \mu \mathrm{L}$ flow 
cell. The absorbance was measured at $532 \mathrm{~nm}$ (the mobile phase consisted of 300 $\mathrm{mL} / \mathrm{L}$ methanol in $50 \mathrm{mmol} / \mathrm{L}$ potassium dihydrogen phosphate buffer, $\mathrm{pH}$ 7.0). 1, 1, 3, 3-tetraethoxypropane was used as a standard, and MDA-TBA reactive substances' values were expressed as MDA (mmol/L). The HPLC system consisted of a solvent delivery pump (JASCO 980-PU, Tokyo, Japan), a reversed-phase column (Luna C18, $250 \mathrm{~mm} \times 4.6 \mathrm{~mm}$, Phenomenex, CA, USA), and a UV-Vis detector (Jasco, UV-975, Tokyo, Japan) operated at $532 \mathrm{~nm}$.

Statistical analyses The results were analysed using SPSS (version 21.0) software. The data are presented as a mean \pm standard error of mean (SEM). Residuals were tested for normality using the Kolmogorov-Smirnov method. Both parity (multiparous vs primiparous) was included in initial models, and body condition score was included as a covariate. Body condition score did not significantly affect any of the models, and was excluded from further analysis. Statistical analysis was performed using one-way ANOVA, followed by Bonferroni-corrected post-hoc comparisons. Effect sizes (Partial-eta ${ }^{2} ; \eta^{2} \mathrm{p}$ ) are reported for all significant ANOVAs. The alpha level for all statistical tests was set at $P<0.05$.

\section{Results}

There was a significant main effect of group on total antioxidant capacity (TAC), $F$ $(4,64)=3.35, P=0.02, \eta^{2} p=0.17$. Bonferroni-corrected post-hoc tests revealed that there were no detectable significant differences between the treatments at the adjusted alpha-level, but there was a suggestion of a difference between groups $M$ $(446.44 \pm 23.75)$ and $C(550.55 \pm 28.41)(P=0.056)$. There were no main effects of group on malondialdehyde (MDA) $(F<1)$, nor total thiols $(F<1)$. 
With the inclusion of multiparity in the model, a significant effect of group on TAC resulted for groups $E, M$ and $L ; F(2,43)=3.8, P=0.03, \eta 2 p=0.15$."

Figure 1 displays the data for TAC according to group with parity included in the model. For TAC, including parities in the model for groups $E, M$ and $L$ revealed a significant effect of group, $F(2,43)=3.8, P=0.03, \eta^{2} p=0.15$. Here, the Bonferronicorrected post-hoc test revealed group $\mathrm{M}<$ group $\mathrm{L}(P<0.03)$. In addition, there was a significant main effect of number of pregnancies on TAC, with primiparous < multiparous, $F(1,43)=4.46, P<0.05, \eta^{2} p=0.09$. There was no group $\times$ parity interaction $(F<1)$. For MDA, there was no effect of group $(F<1)$ nor parities $(F<1)$ nor their interaction, $F(2,43)=2.04, P=0.14$. Finally, there was no main effect of group on total thiols $(F<1)$. There was, however, a main effect of parities, with multiparous cows $(13.27 \pm 0.82)$ having lower total thiols than primiparous cows (15.63 \pm 0.73$), F(1,43)=4.63, P<0.05, \eta^{2} \mathrm{p}=0.1$.

\section{Discussion}

In the present study, cows in the dry period (C group) had the highest level of TAC and postpartum and high yielding cows ( $E$ and $M$ groups, respectively) had the lowest levels of TAC when controlling for parities. Primiparous cows have lesser TAC when compared with multiparous cows, supporting Albera and Kankofer, (2010), who found significantly higher TAC values in plasma of second lactation cows. Social and maintenance behaviour of primiparous and multiparous cows were compared by González et al. (2003). They concluded that cows giving birth for the first time had to face more intensive stress not only because of new physiological experience but also because of new procedures in herd management. Based on our data, it appears that 
multiparous cows may become accustomed to diverse circumstances more readily than primiparous cows (Liesegang et al. 2007).

. Immediately after parturition, MDA levels increased, while blood TAC and total thiols began to decrease. The main source of plasma total thiols is albumin, which is synthesized in the liver. Lower plasma total thiols levels observed in the $E$ and $M$ groups might explain by the reduction in liver function that is usually observed in the early postpartum period (Celi, 2010). Lower levels of total thiols was seen in multiparous cows compared to primiparous cows. Increase of metabolic rate after calving in accordance with increase of milk production in multiparous cows might be responsible of the elevation of respiratory electron transfer and use of total thiols as efficient antioxidants for reacting with free radicals to protect against damages (Rahman, 2007).

MDA is an end product formed via the decomposition of certain primary and secondary lipid peroxidation (Janero, 1990). In the present study, comparison among different groups and between multiparous and primiparous cows found no differences in MDA level. Despite there being no detectable differences between the oxidative measures in the groups, we reasoned that variation in oxidative parameters may be dependent on cumulative impacts of multiple pregnancies in groups $\mathrm{E}, \mathrm{M}$ and $\mathrm{L}$. Previous studies in dairy cows have found contrasting results; some findings show dramatic changes in plasma MDA concentrations (Bernabucci et al. 2005), whilst others don't show no significant changes (Castillo et al. 2005; 2006). Discrepancies are due to the great individual variations in MDA concentrations measured in the various studies or different methodologies employed to assess MDA (Castillo et al. 
2005, 2006; Celi, 2011). Similarly, studies in transported cattle have failed to report a consistent change in MDA concentration (Celi, 2011). It should also be mentioned that in the present study, all the animals were clinically healthy. Our results reveal for the first time that the increase in TAC is not parallel to an increase in MDA levels especially in primiparous cows. This means that the metabolism of these cows produces peroxides faster than the capacity of their antioxidant system.

\section{Conclusion}

Any attempts to reduce prevalence of production diseases should rely on a continuous and comprehensive monitoring of appropriate indicators on the farm level and accounting the rate of ability of dairy cows in different stages of lactation to cope with unbalanced situations. Knowing the weakness in some parts, can help us to support those cows. Many studies revealed consumption of antioxidants and induction of oxidative stress in disease and disorders conditions. The present study demonstrates for the first time that apparently clinically healthy dairy cows may have lower total antioxidant capacity (especially in primiparous cow) and that this condition might make them sensitive to stress, disease or health problems. Furthermore, we suggest that measurement of total antioxidant capacity maybe a useful addion to traditional metabolic profile tests for evaluating the herd health status.

\section{Acknowledgements}

The authors would like to acknowledge the University of Birjand for support of this study. Also we highly appreciate of professor Zarban for measurement of laboratory parameters and staff of Animal Health Management Department of Shiraz University for their valuable comments. 


\section{References}

Albera E \& Kankofer M 2010 The comparison of antioxidative/oxidative profile in colostrum, milk and blood of early post-partum cows during their first and second lactation: Reproduction in Domestic Animals 45 417-425

Bernabucci U, Ronchi B, Lacetera N, \& Nardone A 2005 Influence of body condition score on relationships between metabolic status and oxidative stress in periparturient dairy cows: Journal of Dairy Science 88 2017-2026

Castillo C, Hernandez J, Bravo A, Lopez-Alonso M, Pereira V \& Benedito JL 2005 Oxidative status during late pregnancy and early lactation in dairy cows: The Veterinary Journal 169 286-292

Castillo C, Hernandez J, Valverde I, Pereira V, Sotillo J, Alonso ML \& Benedito JL 2006 Plasma malonaldehyde (MDA) and total antioxidant status (TAS) during lactation in dairy cows: Research in Veterinary Science 80 133-139

Celi P 2010 The role of oxidative stress in small ruminants' health and production:

Revista Brasileira de Zootecnia 39 348-363

Celi P 2011 Biomarkers of oxidative stress in ruminant medicine: Immunopharmacology and Immunotoxicology 33 233-240

Del Rio D, Stewart AJ \& Pellegrini N 2005 A review of recent studies on malondialdehyde as toxic molecule and biological marker of oxidative stress: Nutrition, Metabolism and Cardiovascular Diseases 15 316-328

González M, Yabuta AK \& Galindo F 2003 Behaviour and adrenal activity of first parturition and multiparous cows under a competitive situation: Applied Animal Behaviour Science 83 259-266

Halliwell B 2006. Reactive species and antioxidants. Redox biology is a fundamental theme of aerobic life: Plant Physiology 141 312-322

Halliwell B \& Gutteridge JM 1999 Free radicals in biology and medicine (Vol. 3, pp. 1543). Oxford: Oxford university press. 
Hu ML 1994 Measurement of protein thiol groups and glutathione in plasma: Methods in Enzymology 233, 380-385

Janero DR 1990 Malondialdehyde and thiobarbituric acid-reactivity as diagnostic indices of lipid peroxidation and peroxidative tissue injury: Free Radical Biology and Medicine 9 $515-540$

Kolver ES \& Macmillan KL 1994 Variation in selected blood plasma constituents during the post-partum and breeding periods in dairy cows: New Zealand Veterinary Journal 42 $161-166$

LeBlanc S 2010 Monitoring metabolic health of dairy cattle in the transition period: Journal of Reproduction and Development 56 29-35

Liesegang A, Risteli J, \& Wanner M 2007 Bone metabolism of milk goats and sheep during second pregnancy and lactation in comparison to first lactation: Journal of Animal Physiology and Animal Nutrition, 91 217-225

Macrae Al, Whitaker DA, Burrough E, Dowell A \& Kelly JM 2006 Use of metabolic profiles for the assessment of dietary adequacy in UK dairy herds: The Veterinary Record $159655-661$

Mandebvu P, Castillo JB, Steckley DJ \& Evans E 2003 Total antioxidant capacity: A tool for evaluating the nutritional status of dairy heifers and cows: Canadian Journal of Animal Science 83 605-608

Payne JM, Dew SM, Manston R, \& Faulks M 1970 The use of a metabolic profile test in dairy herds: Veterinary Record 87 150-157

Piccione G, Borruso M, Fazio F, Grasso F \& Caola G 2006 Oxidative stress evaluation during milking period in the ewes: Journal of Applied Animal Research 29 109-112

Poljsak B, Šuput D, \& Milisav I 2013 Achieving the balance between ROS and antioxidants: when to use the synthetic antioxidants: Oxidative Medicine and Cellular Longevity 1-11 


\section{Pregel P, Bollo E, Cannizzo FT, Biolatti B, Contato E \& Biolatti PG 2005}

Antioxidant capacity as a reliable marker of stress in dairy calves transported by road:

Veterinary Record 156(2) 53-54

Rahman K 2007. Studies on free radicals, antioxidants, and co-factors: Clinical Interventions in Aging 2 219- 236

Sepúlveda-Varas P, Huzzey JM, Weary DM \& Von Keyserlingk MAG 2013 Behaviour, illness and management during the periparturient period in dairy cows: Animal Production Science 53 988-999

Zdunczyk Z, Flis M, Zielinski H, Wróblewska M, Antoszkiewicz Z \& Juskiewicz J 2006 In vitro antioxidant activities of barley, husked oat, naked oat, triticale, and buckwheat wastes and their influence on the growth and biomarkers of antioxidant status in rats: Journal of Agricultural and Food Chemistry 54 4168-4175 
Tables

Table 1: The composition of the diets of different groups (\% DM)

\begin{tabular}{lllllllll}
\hline Feed/Group & E1 & E2 & M1 & M2 & L1 & L2 & F & C \\
\hline Alfalfa hay & 21.7 & 23.5 & 21.4 & 23.8 & 24.2 & 20.9 & 19.1 & 21.0 \\
Corn silage & 18.2 & 18.6 & 20.4 & 16.4 & 18.7 & 19.3 & 46.6 & 31.1 \\
Concentrate & 60.1 & 57.9 & 58.2 & 59.8 & 57.1 & 55.3 & 26.5 & 44.3 \\
Wheat bran & - & - & - & - & - & 4.5 & 7.8 & 3.6 \\
\hline
\end{tabular}

Group E: dairy cows from the 3th to 21th day of lactation, (E1: primiparous, E2: multiparous); Group M: dairy cows from the 45th to 100th day of lactation, (M1: primiparous, M2: multiparous); Group L: dairy cows from the 260th day of lactation until the end of milk production, (L1: primiparous, L2: multiparous); Group F: dairy cows in the far-off dry period (from the 305th -345th day); Group C: dairy cows in the close-up dry period (from the 346th-360th day).

Table 2: Estimated daily nutrients fed to the cows in different groups

\begin{tabular}{ccccccccc}
\hline Feed/Group & E1 & E2 & M1 & M2 & L1 & L2 & F & C \\
\hline DM (kg/day) $^{\mathrm{a}}$ & 24.7 & 26.8 & 24.4 & 28.2 & 21.4 & 20.7 & 11.8 & 12.9 \\
NEL(Mcal/kg DM) & 1.66 & 1.65 & 1.66 & 1.7 & 1.65 & 1.54 & 1.48 & 1.59 \\
CP (\%DM) & 15.2 & 15.3 & 15.4 & 15.2 & 15.0 & 13.5 & 13.1 & 15.1 \\
RDP (\%CP) & 62.4 & 61.8 & 62.5 & 63.4 & 62.4 & 65.1 & 69.6 & 69.5 \\
RUP (\%CP) & 37.6 & 38.2 & 37.5 & 36.6 & 37.8 & 34.9 & 30.4 & 30.5 \\
NDF (\%DM) & 32.6 & 33.2 & 32.5 & 31.8 & 33.6 & 36.3 & 44.8 & 36.8 \\
NFC (\%DM) & 41.4 & 40.8 & 41.1 & 41.9 & 40.9 & 42.6 & 35.0 & 41.3 \\
EE (\%DM) & 5.0 & 5.0 & 5.0 & 5.1 & 4.7 & 2.7 & 3.2 & 3.7 \\
Ca (\%DM) & 0.91 & 0.93 & 0.94 & 0.90 & 0.90 & 0.80 & 0.51 & .053 \\
P (\%DM) & 0.40 & 0.41 & 0.41 & 0.39 & 0.38 & 0.36 & 0.47 & 0.42 \\
\hline
\end{tabular}

a Dry matter (DM), net energy lactation (NEI), crude protein (CP), rumen degradable protein (RDP), rumen undegraded protein (RUP), neutral detergent fiber (NDF), non-fibrous carbohydrates (NFC), ether extract (EE). Group E: dairy cows from the 3th to 21th day of lactation, (E1: primiparous, E2: multiparous); Group M: dairy cows from the 45th to 100th day of lactation, (M1: primiparous, M2: multiparous); Group L: dairy cows from the 260th day of lactation until the end of milk production, (L1: primiparous, L2: multiparous); Group F: dairy cows in the far-off dry period (from the 305th -345th day); Group C: dairy cows in the close-up dry period (from the 346th-360th day). 
Figures
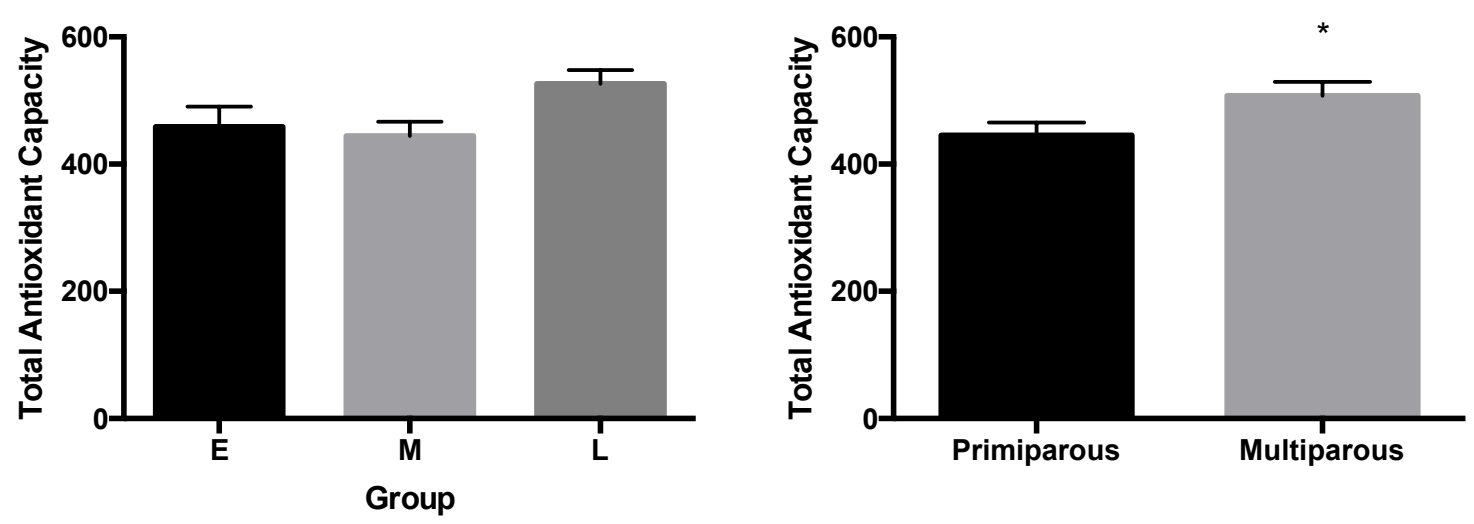

Figure 1. Total antioxidant capacity ( $\mathrm{mmol} / \mathrm{L}$ ) for cows in groups $\mathrm{E}, \mathrm{M}$ and $\mathrm{L}$, and for primiparous and multi parous cows; Group E (fresh): dairy cows from the 3th to 21th day of lactation, Group M (peak milk): dairy cows from the 45th to 100th day of lactation, Group L: dairy cows from the 260th day of lactation until the end of milk production; Note: ${ }^{*} P<0.05$ (Bonferroni corrected). 